\title{
VIRAL AND BACTERIAL ZOONOTIC DISEASES
}

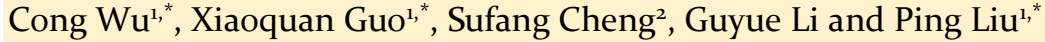

'Jiangxi Provincial Key Laboratory for Animal Health, Institute of Animal Population Health, College of Animal Science and Technology, Jiangxi Agricultural University, Nanchang, Jiangxi, China

${ }_{2}^{2}$ Jiangxi Biological Vocational College, Nangchang City, Jiangxi Province, China

*Corresponding author: pingliujx@163.com (P Liu); wucong0451@outlook.com (C Wu); xqguo20720@aliyun.com (X Guo)

\section{INTRODUCTION}

Zoonotic diseases are infectious diseases that can spread from animals to humans. In this chapter, description about zoonotic diseases in different animals, including companion animals (Section 1), ruminants (Section 2), poultry (Section 3), equines (Section 4) and swine (Section 5) has been given. For each disease, available information about its characteristics, etiology, epidemiology, pathology, diagnosis, histology, treatment, and prevention has been discussed.

\section{Zoonotic Diseases in Companion Animals}

Companion animals are animals living with us in our homes. These are mostly cats and dogs. These animals satisfy our emotional needs and help us to enjoy a happy and healthy life. It is important to take care of these animals and protect them from different diseases. Therefore, it is especially crucial to prevent and treat zoonotic diseases to protect the family with animals and humans. In this section, description about three zoonotic diseases in companion animals: rabies, leptospirosis and tetanus, has been given.

\section{Rabies}

Rabies is caused by lyssaviruses (rabies virus) and can affect any mammalian species. Dogs are major reservoirs of this disease. It is a fatal disease and can spread to people and companion animals after a potential rabies exposure, such as a bite or scratch by an animal with rabies. The virus can cause brain lesions, leading to death of a person without appropriate care after rabies exposure. It is fatal once the symptoms show up. However, it is preventable by vaccinating dogs and treating patients appropriately after rabies exposure.

\section{Etiology}

Lyssaviruses, such as rabies virus and Australian bat lyssaviruses, are the cause of rabies. The virus has helical symmetry so that viral particles are approximately cylindrical in shape. It is an RNA genome, with negative sense and single-strand. It encodes five conserved genes: These genes are nucleoprotein, phosphoprotein, matrix protein, glycoprotein, and the viral RNA polymerase (Finke and Conzelmann 2005).

\section{Epidemiology}

Rabies virus causes approximately 59,000 deaths worldwide each year, and almost all due to dog bites and mostly in Asia and Africa (Hampson et al. 2015). Although rabies virus can live in a range of animals, dogs are the principal host. Although strategies to control rabies, including vaccination of cats and dogs and elimination of stray dogs, have proven to be successful especially in developed countries, dog rabies is still commonly found in many countries especially in developing ones (Wallace et al. 2020).

Elimination of rabies in developed countries has proved to be successful. Most rabies deaths are from countries with poor resources in public health, limited surveillance and preventive treatment (Taylor and $\mathrm{Nel}$ 2015). Vaccination of domestic dog is less effective in rural regions compared with urban regions.

\section{Symptoms}

Animals infected with rabies typically show symptoms of central nervous system disturbance. Common symptoms of rabies are behavioral changes (irritability, hyperexcitability, loss of appetite). Unexplained paralysis appears and worsens as the disease progresses.

Rabies has two forms. The first form is called as "maddog" or furious form. In this form,the animal is irritable, and uses its teeth and claws in an aggressive way with loss of fear of other animals and dilated pupils. Lack of muscle coordination and seizure may be observed. Progressive paralysis finally results in death (Beeler and Ehnert 2020). The second form is called as "dumb rabies" or paralytic form, in which affected dogs show paralysis of the jaw or throat, are unable to swallow and have excess salivation. Dogs seldom attempt to bite and do not show viciousness. Death typically occurs within a few hours after progressive paralysis of the whole body (Alello et al. 2018).

\section{Diagnosis}

Animals affected with rabies may show a range of clinical signs, including aggression, fearfulness, excessive drooling, seizures and paralysis. Multiple tests can be applied to diagnose the disease. Testing methods include reverse transcription (RT) after PCR, testing for antibodies to rabies virus, and examination of rabies 

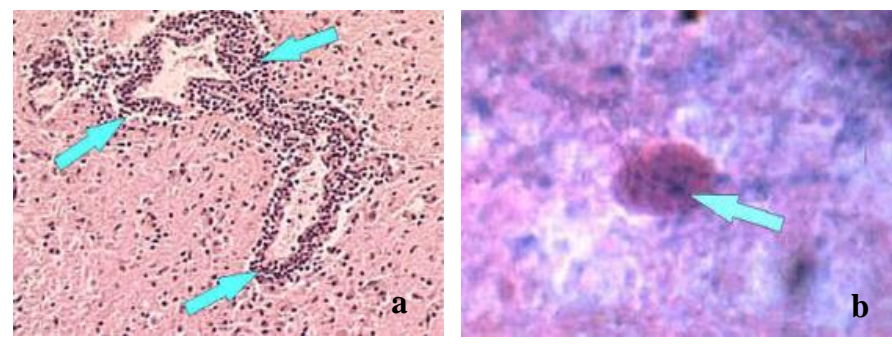

Figure 1: Histopathologic evidence of rabies in brain tissue. Perivascular cuffing or inflammation around a blood vessel in brain tissue with hematoxylin \& eosin staining under 10ox magnification (1a). A Negri body in brain tissue with Sellers staining (1b). Courtesy of Centers for Disease Control and Prevention (2021).

antigen in the nerves. Detection of rabies virus from the brain tissue of affected animals confirms the diagnosis (Centers for Disease Control and Prevention 2021).

\section{Histology}

Samples of brain tissues of the infected animals are stained with hematoxylin and eosin (H\&E). Histologic examination can be performed after the staining. Histological findings in the brain tissue supporting rabies are mononuclear infiltration, perivascular cuffing of lymphocytes or polymorphonuclear cells, Negri bodies, babes nodules consisting of glial cells, and lymphocytic foci (Centers for Disease Control and Prevention 2021). In Fig. 1a, perivascular cuffing around a blood cell is observed in brain tissue with H\&E staining. In Fig. $1 b$, Negri body in infected neuron is observed in brain tissue with Sellers staining (Centers for Disease Control and Prevention 2021).

\section{Prevention and control}

Despite nearly $100 \%$ fatality rate after the appearance of symptoms, rabies is still preventable. Large-scale vaccination of dogs is the most effective strategy in controlling rabies. Other strategies include, elimination of stray dogs, and registration of dog licensing (Banyard and Fooks 2020). Extensive wound cleansing (washing for at least 15 minutes with water, soap and substances that can kill the virus) and injecting effective rabies vaccine after rabies exposure is crucial and can prevent rabies onset and death (World Health Organization 2018).

\section{Leptospirosis}

Leptospirosis is a zoonotic disease, caused by the bacterium Leptospira. Patients affected with leptospirosis can show mild symptoms like headaches and muscle pains, and severe symptoms like lung bleeding or meningitis.

\section{Etiology}

Leptospirosis is caused by Gram-negative bacterium Leptospira. It is an aerobic, 6-20 $\mathrm{mm}$ long and righthanded helical (Karpagam and Ganesh 2020). Leptospira can be classified according to their serovar. More than
250 pathogenic serovars and 24 subgroups of Leptospira have been recognized (Cerqueira and Picardeau 2009). Leptospira lives in the kidneys of affected animals. After being infested, Leptospira enters and moves in the bloodstream and lodges in kidneys. The bacteria are then shed in the urine, without inducing serious ill effect to the animal. Leptospira can be killed at a temperature of $50^{\circ} \mathrm{C}$, or inactivated by ethanal $(70 \%)$, detergents, formaldehyde, and $1 \%$ sodium hypochlorite.

\section{Epidemiology}

Leptospirosis is a worldwide zoonotic infection, with the organisms are found in ponds, rivers and moist soil (Karpagam and Ganesh 2020). Leptospira are found mostly in mammals. There are a range of transmission mechanisms. Leptospira can spread through the urine of the infected animals. It can enter into soil and water and survive for months. Animals in contact with contaminated urine, water or soil can be infected. The bacteria enter the body of the host through mucous membranes (eyes, nose or mouth) or skin. Infected animals shed the bacteria in their urine for years. Leptospirosis is associated with rainfall amount, so the disease spread is seasonal and becomes widespread after heavy rains (Karpagam and Ganesh 2020).

\section{Symptoms}

Patients infected with Leptospirosis show a range of nonspecific symptoms, including fever, vomiting, depression, diarrhea and stiffness. In general, younger animals exhibit more severe symptoms than adults. Cats are less susceptible to Leptospirosis compared with other animals. Cats infected with leptospira may have antibodies detected but show no or mild symptoms (Murillo et al. 2020).

\section{Diagnosis}

Patients infected with Leptospirosis may show increased white blood cell count, blood urea and creatinine, and decreased platelet count and sodium level in the blood (Haake and Levett 2015). ELISA can be used to quantify the IgM antibodies of Leptospira for quick detection of the disease by using L biflexa antigen. ELISA test is useful for rapid detection in the early stage (Rosa et al. 2017).

Microscopic agglutination test (MAT) can be used to help diagnose Leptospirosis (Guedes et al. 2021). In this test, patient sera (after serial dilutions) are mixed with different serovars of Leptospira and examined the mixture for evidence of agglutination under a microscope (Bennett, Dolin and Blaser 2014). Polymerase chain reaction (PCR) can be used to replicate the leptospira DNA from the samples of serum and urine (Bennett et al. 2014).

\section{Treatment}

Leptospirosis can be treated with antibiotics. Dogs affected with Leptospirosis have been recommended to be treated with penicillin derivatives and doxycycline. 
Initial path should be use of doxycycline parenterally because dogs often show symptoms of vomiting. After these symptoms resolve, oral doxycycline can be administered for two weeks to clear leptospires from the renal tubules. Supportive care can be provided for dogs with Leptospirosis, depending on the symptoms and severity of the disease. Supportive care includes maintaining adequate hydration with intravenous fluid therapy, nutrition support, and pain relievers.

\section{Prevention and control.}

Prevention and control measures of Leptospirosis include (1) vaccination of pets, (2) control of rodents (rates, mice, etc), and (3) keeping pets away from contaminated water, animal tissues and urine.

\section{Tetanus}

Tetanus toxemia is a bacterial infection, characterized by muscle spasms. It is caused by the neurotoxin emitted by Clostridium tetani in necrotic tissues. Most mammals (domestic and wild animals) are susceptible to the disease. Cats and dogs are more resistance to the toxin compared with other mammals (Acke et al. 2004).

Clostridium tetani enters the body through wounds, particularly wounds with deep puncture. The bacteria replicate and produce the toxin whichcauses convulsions (Kahn 2007).

\section{Etiology}

Clostridium tetani is an anaerobic bacterium, and can thrive in the environment lacking in oxygen. Potent neurotoxin produced by the bacteria can be absorbed by the motor nerves. It can spread along the nerve tract to reach the spinal cord (Alello et al. 2018). Clostridium tetani is found in soil. It is more common in soil with more organic matter in damp climates. It is commonly found in the feces of many animals including cats and dogs, so that $C$. tetani spores have been reported in manure-treated soils (Acke et al. 2004).

\section{Symptoms}

Incubation period of the disease usually averages 10-14 days but can vary from 1 to several weeks (Alello et al. 2018). Localized stiffness near the wound is firstly observed. One day later, general stiffness shows up with more apparent spasms (Acke et al. 2004). Animals infected with tetanus show increased intensity in reflexes and more violent general spasms. Lockjaw (difficulty in eating food due to spasms of head muscles) is a characteristic symptom. General spasms can hamper respiration and circulation. In dogs and cats, more localized symptoms are seen, because they have high resistance to the toxin (Alello et al. 2018).

\section{Diagnosis}

Diagnosis of tetanus is based on symptoms and history of recent trauma. The diagnosis is confirmed by identifying characteristic spastic paralytic signs. Presence of a wound provides evidence supporting tetanus. Electromyography can also be used to support the diagnosis (Popoff 2020). Evidence of tetanus toxin in serum would confirm the diagnosis (Alello et al. 2018).

\section{Treatment and control}

Patient in the early stages of tetanus may be treated with tetanus antitoxin and tranquilizers. Supporting therapy of tetanus includes cleaning the wounds and administering broad-spectrum antibiotics, including penicillin (Alello et al. 2018). Vaccination with tetanus toxoid can control tetanus. Animals should be injected with another dose of toxoid to increase antibody titer after a dangerous wound is found. Animals surviving tetanus should still be vaccinated with tetanus toxoid, since immunity built may not be adequate (Kahn 2007).

\section{Zoonotic Diseases in Ruminants}

\section{Bovine tuberculosis}

Tuberculosis (TB) is an infectious disease, caused by bacilli of the genus Mycobacterium. In most cases, TB is a chronic debilitating infectious disease, though the progression of the disease may occasionally be very rapid. All species of vertebrates can be infected with the disease (Mahmood et al. 2014). Bovine tuberculosis (Bovine TB) is a zoonotic disease caused by Mycobacterium bovis (Khan and Khan 2007). Bovine TB is especially significant in countries which are not well industrialized and remains a serious problem in many developing countries (Azami and Zinsstag 2018). Cattle are the major reservoir of Mycobacterium bovis. However, this organism has also been isolated from many other species, such as bison, buffaloes, goats, sheep, pigs, deer, dogs, cats, and equines. Cattle-human infection route is the major source of infection to humans. The usual clinical signs of Bovine TB include weakness, fluctuating fever, diarrhea, weight loss, appetite loss, enlarged lymph nodes, and pneumonia (Bapat et al. 2017), which eventually leads to death. Bovine TB is characterized by nodular granulomas and caseous calcified necrotic lesions in tissues and organs (Moustakas et al. 2018).

\section{Etiology}

There are three main types of Mycobacterium tuberculosis: Mycobacterium bovis (bovine), Mycobacterium tuberculosis (human) and Mycobacterium avium (avian). The morphology of Mycobacterium tuberculosis is slightly different in different types. Human tuberculous bacilli are straight or slightly curved elongated bacilli, single or parallel together, mostly sticklike, between branching (Moustakas and Evans 2016). Bovine tuberculosis bacterium is shorter and thicker than that of human tuberculosis, and the pigmentation is uneven. Avian tuberculosis bacterium is short and small, and shows pleomorphic features. This bacterium does not 
produce spores and capsules, nor can it move. It is a gram positive bacterium. Mycobacterium tuberculosis is a strict aerobic bacterium, and the optimum $\mathrm{pH}$ for growth is 5.9 to 6.9 for bovine Mycobacterium, 7.4 to 8.o for the human Mycobacterium and 7.2 for avian Mycobacterium. The optimum temperature for growth is $37-38^{\circ} \mathrm{C}$ (Gilbert et al. 2005).

\section{Epidemiology}

Tuberculosis is the main source of infection. The disease spreads by the pollution of the surrounding environment because the causative organisms can be excreted in feces, milk, urine, and tracheal secretions. It is mainly transmitted through the respiratory and digestive tracts (Khan et al. 2019). Darkness, dampness, dirty environment, excessive labor and milking, and poor feeding can promote the occurrence and transmission of the disease.

\section{Clinical symptoms}

The incubation period of Tuberculosis is generally 10-15 days, but can be up to several months. The disease is chronic, showing progressive wasting, cough and dyspnea. The symptoms are different when the bacteria invade different organs. In cattle, the bacteria mainly invade the lungs, breast, intestine and lymph nodes. The invasion of the bacteria into lungs leads to tuberculosis. The disease is responsible for progressive emaciation of cattle. A short dry cough occurs in the beginning, and it gradually develops into wet cough. Grinding sounds could be heard in the pleural tuberculosis. The invasion of bacteria into the breast leads to mammary tuberculosis. In this condition, milk production decreases gradually with pus sometimes found in the milk. The invasion of organisms into lymph nodes leads to lymphatic tuberculosis, in which lymph nodes are enlarged. It is commonly found in the mandible, pharyngeal, neck and inguinal lymph nodes. The invasion of bacteria into the intestine is most common in calves and characterized by alternating constipation and dysentery or by intractable dysentery.

\section{Pathological changes}

The lesions of tuberculosis are characterized by the formation of white tuberculous nodules in the lungs and other affected tissues. The nodules are off-white, translucent and hard. The pearly-like nodules in the pleura and peritoneum are commonly known as "pearl disease". In late stages of the disease, there is caseous necrosis or calcification in the center of the nodule, or the formation of pus cavity (Dallenga et al. 2017). Histopathological examination shows a large number of Mycobacterium tuberculosis in the nodules. Microscopic examination of lungs usually reveals macro and micro tubercles, with both caseating and non-caseating centers (Mahmood et al. 2014). Extensive caseous material arranged in concentric lamellar fashion is observed in mature tubercles (Figs. 2 and 3). These tubercles are further enclosed within the fibroblastic rim punctuated by lymphocytes, plasma cells, macrophages and massive population of multinucleated giant cells (Figs. 4 and 5). Eosinophilic homogeneous cellular fluid can be seen in the alveoli. Abundant rod shaped bacilli can also be visualized (Mahmood et al. 2014).

\section{Diagnosis}

Tuberculosis can be diagnosed on the basis of clinical symptoms, blood tests, X-ray, and tuberculin test (AlZamel 2012). Tuberculin test is a convenient and reliable method.

\section{Prevention and control}

Healthy cattle without tuberculosis should be quarantined once a year in spring and autumn. People with tuberculosis should not be allowed to raise livestock. Feeding and management practices may be improved to ensure environmental hygiene.

\section{Bovine Spongiform Encephalopathy}

Bovine Spongiform Encephalopathy (BSE) is a progressive, fatal neurological disease of adult cattle. It is characterized by mental disorder, ataxia, hypersensitivity to vision, touch and hearing. The histopathological features are spongy vesicular degeneration of the brain (Prusiner 1991).

\section{Etiology}

The pathogen is prion, which is an abnormal protein formed by the normal glycoprotein on the surface of the host nerve cell and mainly composed of $\beta$ folding (Bolton et al., 1982). Prions can survive in the soil for up to three years. Formalin, hydrogen peroxide and phenol cannot deactivate them.

\section{Clinical symptoms}

The incubation period for BSE is normally 4-5 years. The disease is characterized by long incubation period and slow progression. The development of BSE can be divided into four stages: biological onset stage, preclinical stage, clinical stage and transition stage. Cattle infected with BSE can show mental disorders, like fear or become aggressive when approached by persons (Bruce et al. 1994), motor disorders such as ataxia, tremor or collapse and sensory disorders including hypersensitivity to touch, sound, and light. The infected cows may have a gait of "goose step" with limbs over-extended.

\section{Pathological changes}

Histo-pathological changes in BSE are mainly limited to the central nervous system. The main features are: vacuoles degeneration, loss of neurons and glial cells hypertrophy, nerve cells swelling, and cytoplasm shrinkage. 


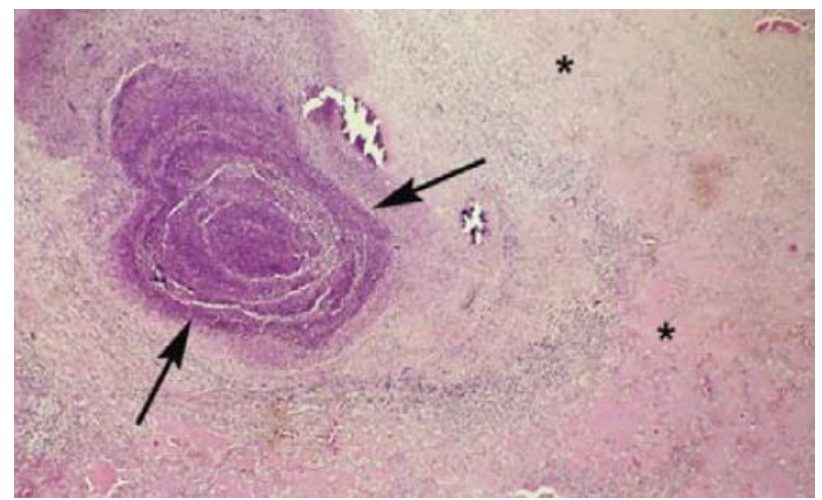

Figure 2: Lungs of a cow died of bovine tuberculosis exhibiting lamellated arrangement of caseous material (arrows) in mature tubercle and edema in alveoli (asterisk). H\&E stain, 10oX. Courtesy of Mahmood et al. (2014).

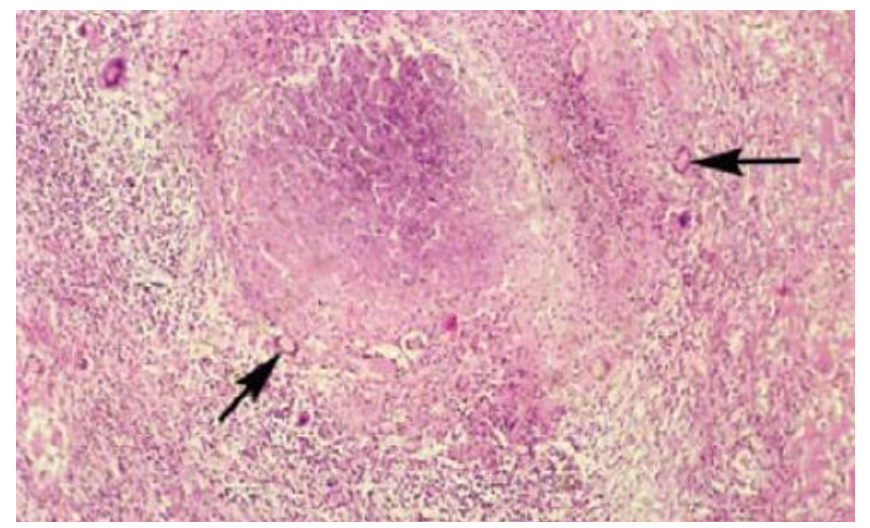

Figure 3: Immature tubercle with large number of multinucleated giant cells (arrows). H\&E stain, 20oX. Courtesy of Mahmood et al. (2014).

In addition, there is obvious degeneration and necrosis of nerve cells. Apoptosis of nerve cells and the formation of vacuolar structures lead to disorders in signal transduction, so that the animals can show autonomic movement disorders, fear, biological clock disorders and other neurological symptoms.

\section{Diagnosis}

The primary diagnostic method of BSE is histopathological examination. Brain tissue sections are inspected under a microscope. The characteristic pathological changes in neurons are more common in the transverse sections of the medulla oblongata, the collateral cerebellar angle, the pons and the midbrain. The occurrence frequency is very high, especially in the nucleus of the solitary tract of the medulla oblongata and the nucleus of the spinal trigeminal nerve. Therefore, the examination of degenerative changes, such as vacuolar degeneration of neurons in the cross section of the brain, is a routine method to diagnose the early onset of BSE (Ducrot et al. 2008).

\section{Prevention and control}

To control BSE, it is important to identify animals

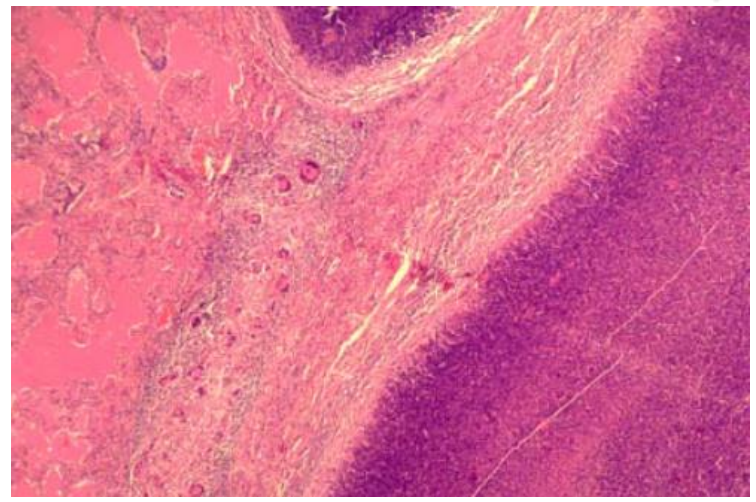

Figure 4: Mature tubercle of lungs exhibiting granular caseous center with punctuation of brim with fibroblasts and massive population of giant cells. H\&E stain, 10oX. Courtesy of Mahmood et al. (2014).

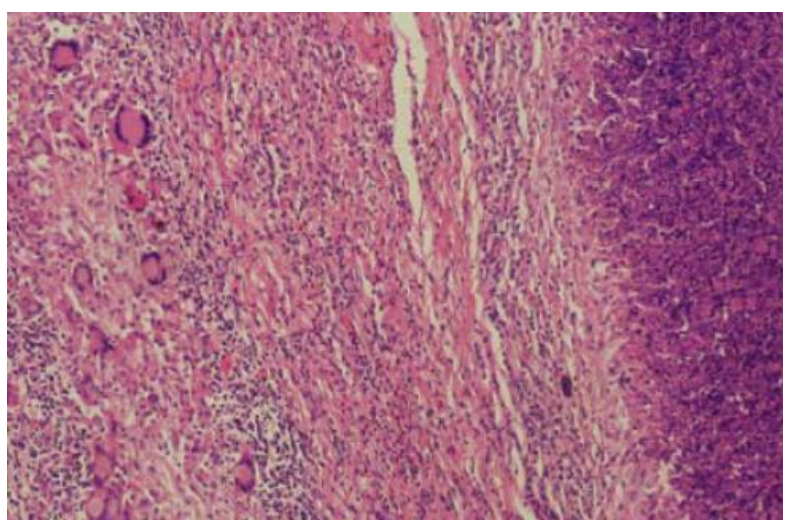

Figure 5: Granular caseation and punctuation of fibroblasts and massive population of giant cells in lungs of a bovine tuberculosis died cow. H\&E stain, 20oX. Courtesy of Mahmood et al. (2014).

infected with BSE. Good feeding management and BSE epidemic prevention can help control BSE.

\section{Zoonotic Diseases in Poultry}

\section{Avian influenza}

Avian flu is a viral zoonosis caused by avian influenza viruses. Avian flu can cause multiple symptoms, especially those of the respiratory tract.

\section{Etiology}

Avian influenza viruses belong to influenza A group of viruses of the orthomyxoviridae family, which in turn belongs to RNA viruses that are easily mutated. Most of the influenza A viruses infect only birds, and a few infect humans and mammals, such as pigs. Influenza B and $C$ viruses have been found in seals and pigs, respectively. Influenza A viruses are pleomorphic, encase with the diameter ranging from $80 \mathrm{~mm}$ to $120 \mathrm{~mm}$. The genome of the virus is a segmented, negative-sense, single-stranded RNA. Because of the different antigenicity of the outer membrane hemagglutinin $(\mathrm{H})$ and neuraminidase $(\mathrm{N})$ proteins, they are divided into $16 \mathrm{H}$ subtypes $\left(\mathrm{H}_{1}-\mathrm{H} 16\right)$ and $9 \mathrm{~N}$ subtypes $\left(\mathrm{N}_{1}-\mathrm{N}_{9}\right)$. The avian influenza virus subtypes that infect humans are mainly $\mathrm{H}_{5} \mathrm{~N}_{1}, \mathrm{H}_{9} \mathrm{~N}_{2}$ and 
$\mathrm{H}_{7} \mathrm{~N}_{7}$, with $\mathrm{H}_{5} \mathrm{~N}_{1}$ being the most virulent, the sickest and the most fatal ( $\mathrm{Wu}$ et al. 2017). Studies have shown that highly pathogenic strain $\left(\mathrm{H}_{5} \mathrm{~N}_{1}\right)$ can be derived from low pathogenic strains of avian influenza $\left(\mathrm{H}_{5} \mathrm{~N}_{2}, \mathrm{H}_{7} \mathrm{~N}_{7}\right.$, $\mathrm{H}_{9} \mathrm{~N}_{2}$ ) after 6 to 9 months of rapid mutation among birds (Quan et al. 2019).

\section{Epidemiology}

Depending on the virulence of the strain, the transmission rate and fatality of the virus vary (Bui et al. 2017). The disease mainly occurs between winter and spring, or earlier between autumn and winter. Sick birds and the birds that carry the virus are the main source of infection of avian flu. In addition, wild birds and pigs can also become the carriers of this disease. Many poultry birds can be infected including turkeys, pigeons, guinea fowl, chickens, ducks, geese and parrots (Peng et al. 2018). Chickens and turkeys are the most susceptible, with high morbidity and mortality rates.

\section{Symptoms}

The symptoms of avian influenza include loss of appetite, head and facial edema, and nervous symptoms. Respiratory symptoms are mainly cough, canthus tears, and sneezing. Sick poultry can show diarrhea, with discharge of yellow green thin feces. There are numerous necrotic nodules. Dark red blood patches can be seen in the hairless parts of sick chickens. The virus can also affect the laying performance of chickens, and decrease the egg production.

\section{Pathological changes}

The sick chickens show head swelling, hairless bleeding patches, oral bleeding and necrotizing lesions. Surface of the heart shows bleeding spots, accompanied by fibrinous pericarditis. Bleeding spots are also seen in adipose tissue around the muscles of stomach and on the surface of the small intestine. The pancreas is enlarged and shows signs of bleeding. There is obvious grayish white exudate under the skin of air bag and oviduct.

\section{Diagnosis}

A preliminary diagnosis of avian influenza can be made on the basis of clinical symptoms of the disease and the characteristics of meteors. To make an accurate diagnosis, laboratory testing methods such as overcomplement binding and hemagglutination inhibition are needed.

\section{Treatment and prevention}

Multi-electrolyte or weak saline is added in the drinking water of birds, and $1 \%$ potassium permanganate can be offered once a week to improve the resistance of poultry for the prevention and control of the disease. Quinarene of sick birds is necessary.

\section{Salmonellosis}

Salmonellosis is caused by the bacterium Salmonella. Every year, a large number of people and livestock suffer from salmonella infection and even death.

\section{Etiology}

Salmonella, belonging to the genus Salmonella, is a gramnegative bacterium with size between $0.6 \times 3.0$ microns. It has no buds. In general, it also has no capsule, except the Salmonella pullorum and Salmonella typhimurium. It mainly survives in the intestinal tract of the host, with weak resistance to heat and can survive for less than 15 minutes at temperatures above $60^{\circ} \mathrm{C}$.

\section{Epidemiology}

Diseased animals and carriers are the main source of Salmonella infection. The causative bacteria can be transmitted through host metabolites and excreta, and infect healthy animals through the digestive tract. It is infectious to all species of poultry and domestic animals. Young animals are more susceptible to the infection than adult animals. The disease has no obvious seasonality, and is generally sporadic or epidemic after its occurrence in herds. In addition, feeding conditions and poor environment, as well as climate and childbirth, can increase the occurrence of this disease.

\section{Symptoms}

There are three manifestations of infection in chicks, namely acute septic type, arthritis type and nervous type. The main characteristics of acute septic type are pullorum and dyspnea, with the mortality rate can reach $100 \%$. Arthritis type is mainly characterized by swelling of the hocks, and the fatality rate is about $30 \%$. The main symptoms of neurologic type are motor dysfunction, including head tilt, head back, beak touch and turning, with the fatality rate is about $10 \%$.

The disease of adult chickens is usually subacute in nature and results in high morbidity rates. The main characteristic is swelling of the synovial sac of the hock and sternum; the fatality rate is about $10 \%$. In adult chickens, symptoms are not obvious, and the infection is usually inconspicuous, sometimes with dysentery and severe stress that results in sepsis and a significant decrease in egg production. The fatality rate is about $20 \%$ (Kariuki et al. 2015).

\section{Pathological changes}

There are necrotic foci in myocardium, lungs, cecum and large intestine, and the liver becomes brittle and swollen. The gall bladder is enlarged with white urate deposits in the ureter. The pathological changes of laying hens are mild, and the main manifestations include liver and kidney swelling, salpingitis and ovaritis. 


\section{Diagnosis}

The clinical symptoms of salmonellosis are similar to those of swine fever, so it is impossible to draw a direct conclusion based on symptoms. A preliminary diagnosis can be made on the basis of clinical symptoms observed in sick animal, and then the feces or secretions are sent for inspection. After collection, samples are inoculated on the SS Agar medium and cultured at $37^{\circ} \mathrm{C}$ for 24 hrs. The characteristics of the bacterial colonies are basically the same as those of Salmonella. The extracted and isolated pathogenic bacteria are further purified, isolated and cultured. Drug sensitivity test has revealed that the isolated pathogens were highly sensitive to cefuroxime, cefotaxime and gentamicin.

\section{Prevention and control}

Combined with drug sensitivity test, the infected chickens can be treated with cefotaxime and gentamicin. Salmonella agglutination test can be used for the diagnosis of the disease. To control the spread of this bacterial disease, it is necessary to guide farmers to strengthen daily quarantine and formulate strict sanitation and disinfection strategies. Vehicles and personnel entering and leaving the farm need to carry out strict sanitation and environmental disinfection. Farms also need to develop strict quarantine inspection system to phase out flocks of chickens.

\section{Zoonotic Diseases in Equines}

\section{Glanders}

Glanders is an infectious disease of equines caused by Burkholderia mallei. It is a highly contagious disease of hoofed animals including horses, mules and donkeys. People can be infected upon contact with sick animals or pathogenic bacteria. The main clinical symptoms are acute fever, cellulitis, necrosis, abscesses in the respiratory tract, skin, muscles and other places, formation of melirax nodules, ulcers and scars in the nasal cavity, larynx, tracheal mucosa or skin, and the occurrence of melirax nodules in the lungs, lymph nodes or other important organs.

\section{Etiology}

The pathogen of glanders is Burkholderia mallei. In 1993, Burkholderia was listed in the genus Burkholderia on the basis of its biological characteristics (Khan et al. 2013). The bacterium is Corynebacterium microcurvae, with different sizes, ranging from 2 to $5 \mu \mathrm{m}$ in length and 0.5 to $1.0 \mu \mathrm{m}$ in width. It is mostly isolated and sometimes arranged in pairs. It has no flagella and cannot move. It has no capsule or spores. It is negative for gram stain. The optimal temperature for growth is $37-38^{\circ} \mathrm{C}$ and $\mathrm{pH}$ is 6.8 7.o. There are two antigens for melioidosis; one is specific antigen, and the other is common with melioidosis antigen. There is cross reaction with melioidoid in agglutination test, complement binding test and allergic reaction. This bacterium does not produce exotoxin. The protein part of its toxin in vivo, mallein, can cause allergic reaction in infected animals, which is used as skin test antigen for diagnosis. The bacteria show strong resistance and can survive for 1 hour in feces and urine, 70 days in water, and 6 months in sterilized tap water. However, it can survive for only 10-15 days in dry environment. Exposure of the organism to direct sunlight for 24 hours and a temperature of $56^{\circ} \mathrm{C}$ for $15 \mathrm{~min}$ can kill it. It can also be killed in 3\% kerosene soap solution, $10 \%$ lime milk and $2 \%$ formaldehyde in 1 hour.

\section{Epidemiology}

Equine gangrene (Glanders) can be transmitted to healthy horses from sick horses. Natural infection is transmitted through nasal secretions, wet cough and pus from ulcers of sick animals. The infection can also occur through the digestive tract when feeding in the same tank, drinking in the same bucket, biting each other and with the intake of feed and drinking water contaminated by anthrax (Van et al. 2013). Infection caused by skin or mucous membrane trauma is rare. The infection of human gangrene is mainly through traumatic skin and mucous membrane, but rarely through food and drinking water. Humans and different animals are susceptible to this disease. Donkeys are the most susceptible animals, followed by mules. Cattle, goats, dogs, sheep and goats are occasionally infected with the disease. The captured wild lions and tigers can die of the disease due to eating the meat of infected animals.

The new disease area often presents this disease as an explosive acute epidemic. In the frequently-affected areas, the horse population shows slow and continuous transmission. Once the disease appears in the absence of timely eradication measures, it can exist for a long time, and most of the cases are chronic or recessive. Stress factors, such as poor management and feeding, overwork, disease or long-distance transportation, can cause an explosive epidemic with a large number of deaths.

\section{Symptoms}

The incubation period of the disease varies from several hours to several months. Clinically, there are two types; acute and chronic. Acute glanders is fatal within a few days to weeks, and begins with chill and high fever, depression, anorexia and emaciation. It is clinically characterized by bouts of acute cellulitis, local swelling, necrosis and ulceration at the site of skin infection, forming an ulcer with irregular edge and gray base, and covered with grayish yellow exudate (D'Elia et al. 2019). The adjacent lymph nodes are swollen, and there are many muscular and subcutaneous nodular abscesses in the lymphatics. When the abscess is opened, the red or gray pus is discharged, the mouth does not heal, and usually forms a fistula. Abscesses also appear in skin or soft tissue, and nearby lymph nodes become swollen. Sometimes, abscesses burst and a large amount of pus 
flows out. Joints, bone marrow, liver, spleen, lungs, eyes and central nervous system can also be involved (Samy et al. 2017). The disease progresses slowly and can last for several months to several years. The patients gradually become thin with cachexia and often die gradually.

\section{Diagnosis}

The causative organism, Burkholderia mallei, penetrates the mucosa and reaches the lymphatic system. As the disease progresses, hematogenous spread occurs. The characteristic lesions of Glanders in equids include granulomata and ulcers in different tissues, nodules and fulminating ulcers on the mucous membranes of the nasal passages, larynx and upper lips (Samy et al. 2017). Bone lesions are observed in mules and humans. Modular foci underneath the pleura and diffuse granulomatous nodules may be found in the lungs. Histologic lesions are observed with granulomatosis in the lungs accompanied by inflammatory elements including macrophages, epithelioid cells and small foci of hemorrhage, edema and aggregation of interlobular and intra-alveolar fibrin (Estes et al. 2010).

\section{Treatment and control}

Horses suffering from Glanders should be isolated and thoroughly disinfected. The abscesses must be opened and drained, but care should be taken to avoid infection spread. Animals serological testing can be conducted prior to cross-regional transport to reduce disease spread risks.

Because $B$. mallei is sensitive to sulfadiazine, sulphathiazole, sulphamethazine, sulphamdimidine, tetracyclines, neomycin, oleandomycin, polymyxin B, erythromycin, kanamycin, sigmamycin and nystatin, they can be used to treat the disease. For example, as shown in Figs. $6 \mathrm{~A}$ and $6 \mathrm{~B}$, horses suffering from Glanders show improved symptoms after intravenous injection (Khan et al. 2013). Figs. $7 \mathrm{~A}$ and $7 \mathrm{~B}$ show the effect of treatment follow-up for six months in a horse (Khan et al. 2013).

\section{Zoonotic Diseases in Swine}

\section{Japanese encephalitis}

Porcine Japanese encephalitis is a mosquito-borne porcine reproductive disorder, caused by the Flaviviridae Japanese encephalitis virus (Leake 1990). It is manifested by miscarriage, stillbirth, mummified fetus in sows, and orchitis in boars. Pigs are the main amplification host and the source of infection of Japanese encephalitis virus. The outbreak of Japanese encephalitis in humans is related to the outbreak of this disease in pigs. Therefore, Japanese encephalitis in pigs also has public health significance. The structure of Japanese encephalitis virus (JEV) is shown in Fig. 8 with the viral particle (A), the E protein structure (B) and the cross-section profile of JEV (C) (Luca et al. 2012).

Japanese encephalitis virus belongs to the Flaviviridae family. It is an RNA virus. The virus particles are spherical
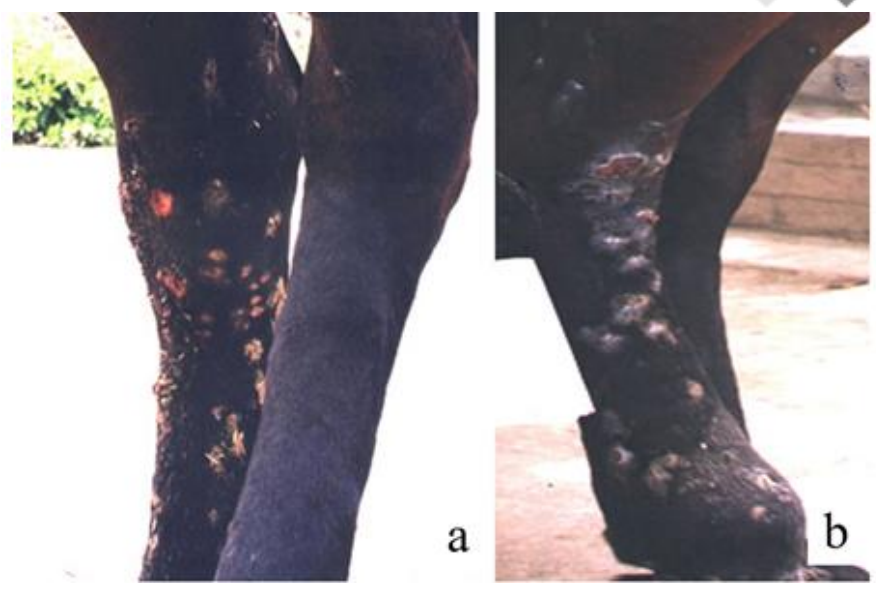

Figure 6: The reaction of experimental treatment to intravenous injection in a horse with Glanders. Ulcer appeared in right hind leg before treatment (a), and healed ulcer and scar after intravenous treatment in the second week (b). Courtesy of Khan et al. (2013).
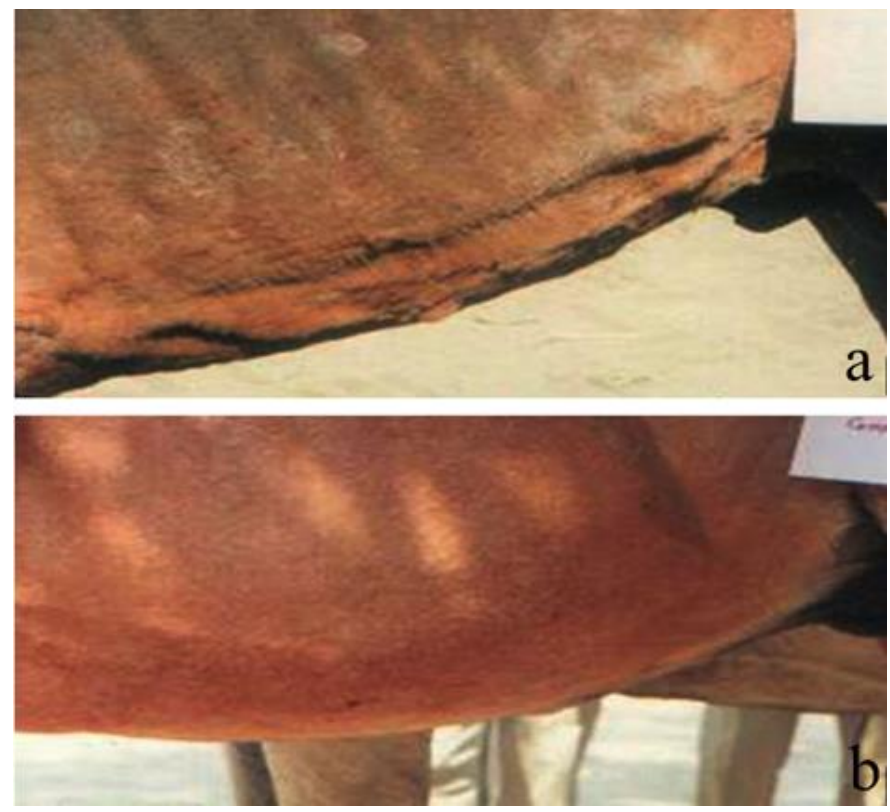

Figure 7: The experimental treatment in the horse with Glanders was followed up for 6 months. Body hair and lateral medial abdominal lymphangitis (a). The lesion (lymphangitis) disappeared completely, the physical condition improved significantly, and the rib boundary disappeared (b). Courtesy of Khan et al. (2013).

in shape with a diameter of about $40 \mathrm{~nm}$. It has a capsule and is sensitive to lipid solvents. It can agglutinate the red blood cells of chickens, pigeons, geese and sheep. A variety of cell monolayers can be used for culture and proliferation of the virus. Chicken embryo culture can also be used. The virus has poor resistance to external environmental factors. Commonly used disinfectants can kill the virus. It can be inactivated by exposure for 30 minutes at $56^{\circ} \mathrm{C}$.

\section{Epidemiology}

Pigs are the main propagation host and source of infection of this virus. Other domestic animals, wild 

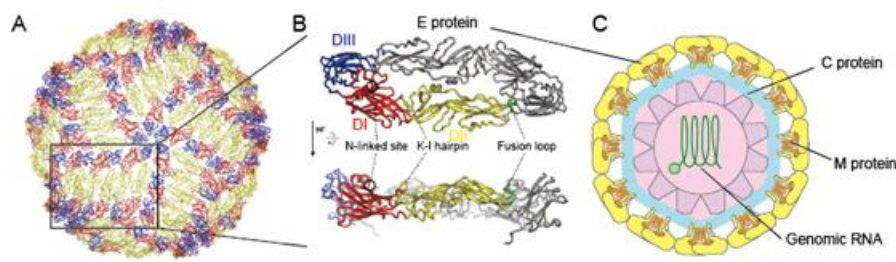

Figure 8: The viral particle (A), the E protein structure (B) and the cross-section profile of Japanese Encephalitis Virus (C). Courtesy of Luca et al (2012).

vertebrates, birds, mosquitoes, amphibians, reptiles, and bats can all serve as the host of Japanese encephalitis virus. Pigs are susceptible regardless of breed and sex, and the age of onset is mostly consistent with sexual maturity. Pigs have a high infection rate, but morbidity rate is low. In addition, humans, equines, cattle and sheep are all susceptible to the infection (Ladreyt et al. 2020).

There is a correlation between porcine Japanese encephalitis and human Japanese encephalitis. The peak of natural infection with Japanese encephalitis virus in pigs occurs 3 to 4 weeks earlier than the peak of infection with human Japanese encephalitis. It spreads through the circulation route of pig-mosquito-human etc. Transmission of the virus can occur between pigs and humans.

The occurrence of this disease is obviously related to the active season of mosquitoes. The virus can often be isolated from blood-sucking insects in endemic areas, especially Culex and Aedes, which are typical source of mosquito-borne infectious diseases. About 90\% of the cases are recorded between July and September, and almost no cases occur from December to April of the following year.

\section{Symptoms}

The incubation period of Japanese encephalitis virus is generally 3 to 4 days. Affected pigs are depressed, and their body temperature rises as high as $40-41^{\circ} \mathrm{C}$, with loss of appetite, thirst and flushing of the eye conjunctiva. The suffering pigs lie down with lethargy, barely stand, and then lie down again. Clinical symptoms also include accelerated heartbeat, slight shortness of breath, cough, dry stool, and dark yellow urine. Some affected pigs have swollen hind limb joints, limp or paralyzed, and have unstable gait.

Pregnant sows often have a sudden miscarriage, with mild food loss or fever before the miscarriage. Miscarriage mostly occurs in the late pregnancy. Symptoms alleviate after miscarriage, and body temperature and appetite return to normal. A small number of sows show discharge of reddish-brown or even gray-brown mucus from the vagina after abortion. After abortion, the rebreeding ability of the sow is not affected. Most aborted fetuses are stillborn, mummified, or dying. Although some of the surviving piglets are normal in appearance, they are weak in physique and cannot stand or suck milk of their mothers. Some piglets also show neurological symptoms after birth, convulsions all over the body, fall to the ground, and die within 1 to 3 days. The survivors vary in size, with most of them weak. In addition to the abovementioned general symptoms, the prominent manifestation in the boar is orchitis after fever. One or both testicles are obviously swollen, which is 0.5 to 1.0 times larger than normal testes. The scrotum folds disappear due to the swelling of the testis. The local temperature is high, with pain. The scrotal skin of the white pig becomes red, the swelling subsides and scrotum returns to normal after 2 to 3 days, or it may become smaller, harder, and loses its function.

\section{Lesions}

Lesions of the Porcine Japanese encephalitis include the endometrial hyperemia and edema of aborted sows, with small hemorrhages and sticky secretions on the mucosa. Scraping of secretions shows mucosal erosion, lower edema, and placenta showing inflammatory reaction. Cerebral edema, congestion of meninges and spinal cord, subcutaneous edema, pleural and abdominal effusion, discoloration of the muscles, with boiled-meat like appearance can be found in aborted fetuses. There are bleeding spots on the serosal membrane, congestion of lymph nodes, necrosis in the liver and spleen, and hypoplasia of the brain and cerebellum in some fetuses. The testicles of the boar are enlarged to varying degree, the testicular parenchyma is congested and shows bleeding spots. Cut surface of testicles shows yellow necrotic foci of varying sizes, surrounded by bleeding spots. The scrotal folds disappear and the scrotum appears shining with large amount of yellow-brown opaque liquid retained in the tunica cavity. In chronic cases, atrophy and hardening of the testicles, adhesion of the testicles to the scrotum and most of the connective tissue can be seen.

\section{Diagnosis}

Preliminary diagnosis of Porcine Japanese encephalitis can be made on the basis of its epidemic characteristics, clinical symptoms and lesion characteristics. For example, the epidemic of this disease has a strict seasonality, and it usually occurs from July to September. The main symptoms in sows are abortion, stillbirth, mummified fetuses, and the one-side enlargement of testicles in boars. However, the diagnosis requires laboratory investigations.

The method of isolation and identification of pathogens is the most classic diagnostic method for Japanese encephalitis. In the early stage of fever, blood and serum can be used to isolate the virus. After the animal dies, brain tissue and body fluids should be taken as soon as possible. Aborted fetuses and placenta can also be used to take samples. The blood sample can be used directly in the experiment. After the brain tissue is ground and homogenized, it is inoculated simultaneously into the brain and subcutaneously in the suckling mice. In recent years, BHK cells have been used for isolation of the virus. After the cells have shown regular lesions, the cell fluid is 
taken to inoculate mice, and the disease is observed. If there is a suspicious disease, the brain tissue is taken and then the virus is identified.

The RT-PCR method mainly amplifies the more conservative $\mathrm{M}$ or $\mathrm{E}$ genes in the genome, which can be used for early pathogen diagnosis. Serological diagnosis can also be conducted. At present, complement fixation test, neutralization test, latex agglutination test, enzymelinked immunosorbent assay, indirect immunofluorescence test, dot immune-filtration detection method and other serological methods are available to detect specific antibodies in the serum or cerebrospinal fluid of patients with Japanese encephalitis (Mansfield et al. 2017). Among these, the hemagglutination inhibition test $(\mathrm{HI})$ is the most commonly used. Because the Japanese encephalitis hemagglutination antibody IgM appears earlier, HI can be used for early diagnosis. The diagnosis can also be made on the basis of a 4 -fold increase in the titer of double serum IgG antibodies. In clinical diagnosis, it should be distinguished from porcine brucellosis, porcine parvovirus disease, porcine pseudorabies, porcine chlamydia, porcine reproductive and respiratory syndrome, and porcine infectious encephalomyelitis.

\section{Prevention}

Preventive strategies of this disease include the management of host animals, with the focus on the management of young animals that have not passed through the summer and autumn seasons and animals introduced from non-epidemic areas. Most of these animals have not been infected with Japanese encephalitis. Once infected, they will develop viremia and become a source of infection. Pigs are proliferation animals of Japanese encephalitis, and sick pigs with this disease should be quarantined immediately.

Another preventive strategy is the elimination of the transmission medium and the control of transmission source. Because mosquitoes are the main transmission vector of the disease, mosquito control measuresshould be strengthened. However, it is difficult to control the epidemic of the disease simply by killing mosquitoes. Therefore, immunization becomes an important preventive measure.

The third preventive strategy is immunization. Currently, a live vaccine of Japanese encephalitis for animals has been developed. In areas where this disease is endemic, immunization of antibody-negative pigs or breeding pigs over 4 months of age one month before mosquito activity, or vaccination one month before mating is advised. A booster vaccination should be given a week later, and then a vaccination is given every year before the start of mosquito season or before breeding.

\section{Listeriosis}

Listeriosis is a food-borne, sporadic zoonotic disease of animals and humans, caused by Listeria mononucleosis, which has a high fatality rate (Lepe 2020). Sick animals are mainly manifested by meningitis, sepsis and pregnancy miscarriage. Affected birds are mainly manifested by necrotizing hepatitis and myocarditis. The disease is widely distributed all over the world.

Listeria monocytogenes is Gram-positive micro-bacteria. Single or two bacteria in the smear are arranged in the "V" shape, without capsules or spores, and with flagella. There are currently 7 serotypes and 16 serovariants. Pigs are more commonly affected by type 1 . The bacteria have strong resistance and can survive in soil and feces for several months. Pasteurization cannot kill these bacteria. However, they can be inactivated after 30-40 minutes exposure at $65^{\circ} \mathrm{C}$. General disinfectants are effective. It is sensitive to streptomycin, tetracycline and sulfa drugs.

\section{Epidemiology}

The range of susceptible animals is very wide and at least 42 species of mammals and 22 species of birds have been proved to be susceptible to this disease. Sheep, cattle, pigs, and rabbits are more susceptible. In poultry, chickens, turkeys, and geese are more commonly affected. Wild animals, wild fowls, and rodents are all susceptible to infection, and they are often the storage hosts of the disease. Humans can also be infected naturally. The feces, urine, milk, mucus and secretions of eyes, nose, and reproductive tract of infected animals and bacteriacarrying animals contain the organism. The disease can be transmitted through the digestive tract, respiratory tract, conjunctiva and injured skin. Contaminated feed and water are the main transmission media, and bloodsucking insects can also transmit the disease. Lack of green feed in winter, sudden changes in weather, internal parasites or salmonella at the time of infection can be the inducement of the disease. The disease is more common in the fertile soil.

The disease is sporadic, with usually only a small number of cases, but the fatality rate is high (Chlebicz and Śliżewska 2018). Although animals of all ages can be infected, the young ones are more susceptible, and the disease is acute in nature. In some areas, the disease occurs mostly in winter and early spring (Belœil et al. 2003).

\section{Clinical symptoms}

The incubation period of the disease is generally 2 to 3 weeks; however, it can be as short as several days, and as long as 2 months. The clinical symptoms are mainly characterized by fever, neurological signs, abortion in pregnant animals, and sepsis in young pigs. The clinical manifestations in different animals are different.

The incubation period of natural infection is usually 2 to 3 weeks, and weaned piglets and suckling piglets show symptoms of meningitis. Body temperature at the beginning of the illness is generally normal, some animals may show low fever; the body temperature can drop below $36.5^{\circ} \mathrm{C}$ in the later stage. At the beginning of the illness, affected animals may show disturbance of consciousness, dyskinesias, circular motions or aimless 
walk, or holding the head against the ground. Some have their heads and necks turned back, with their forelimbs or hind limbs open, showing a typical "star-gazing" posture. In some cases, muscles tremble and become tough, and it is obvious in the neck and cheek muscles. Some animals show paroxysmal spasms, foaming at the mouth, lying on the ground, and swimming-like movements of the limbs. The larger pigs are shaky and show ataxia. Some have paralyzed hind limbs, unable to stand up and drag the floor. Generally, 1-4 days of exhaustion and death are seen, the long course of disease can reach 7-9 days. Piglets often suffer from sepsis, showing increased body temperature, decreased or annulled appetite, cough, diarrhea, rashes, pulmonary edema, dyspnea, blue-purple skin, etc. The course of the disease is 1 to 3 days, and the fatality rate is high. Miscarriages often occur in pregnant sows.

\section{Lesions}

There are usually no obvious gross pathological changes. Diseased animals with neurological clinical symptoms may have changes in meninges with congestion, inflammation and edema in the brain. The amount of cerebrospinal fluid is increased, becomes slightly turbid and contains many cells, and the brainstem becomes soft. The diagnosis depends on histological examination. There are inflammatory changes in the pons and encephalon. Sick animals with sepsis show sepsis changes, small punctate necrosis or multiple abscesses in the liver, spleen, and myocardium, as well as yellow staining of the subcutaneous tissue.

\section{Diagnosis}

Sick pigs with special neurological symptoms show pregnancy miscarriage and increased blood mononuclear cells. Necropsy shows meningeal congestion, edema, and small necrotic foci in the liver. Microscopic examination of brain tissue shows vascular sleeves dominated by mononuclear cell infiltration. A preliminary diagnosis can be made for lesions such as tiny purulent foci, and laboratory tests are required to confirm the diagnosis.

\section{Prevention}

Sanitation, epidemic prevention and breeding management are preventive strategies. Attention should be focused to expelling rodents and other animals with ectoparasites, and not introducing livestock and poultry from epidemic areas. Sick livestock and poultry should be treated in isolation after the onset of the disease, and the livestock houses, cages, utensils, environment and feeding troughs should be disinfected with disinfectants such as bleaching powder, and comprehensive epidemic prevention measures should be taken.

The humans are susceptible to Listeria bacilli, and the symptoms of encephalitis are more common. Workers engaged with diseased livestock and poultry should pay attention to protection and food hygiene. Livestock with
Listeriosis should not be used for food, but they can be allowed to be slaughtered if fully recover from the disease and their health is restored. Listeria monocytogenes is sensitive to some antibiotics, such as sulfa drugs, gentamicin, streptomycin, tetracycline, so they are often used in the prevention and treatment of this disease. But the organism is resistant to penicillin. Early high-dose use of sulfa drugs combined with gentamicin and tetracycline have shown good results. However, in cases with obvious neurological symptoms, treatment is difficult and may not be very effective.

\section{Streptococcosis}

Streptococcosis is a general term for different clinical types of infectious diseases caused by a variety of different groups of streptococci. It is characterized by acute cases with sepsis and meningitis, and chronic cases with arthritis, endocarditis and tissue suppurative inflammation (Alello et al. 2018). Streptococcus is divided into 20 serogroups (Good 2020). Acute cases of this disease are mainly caused by group $C$ streptococci, which has a high incidence and fatality rate and is very harmful. The most common chronic disease is lymph abscess caused by group E streptococcus.

Bacteria of the genus Streptococcus are round or oval, and are often arranged in chains of varying lengths. They often appear as short chains on solid media and long chains on liquid media. They do not form spores and generally have no flagella. Some strains can form capsules in the body or in serum-containing media, and are Grampositive. These bacteria grow better on the medium containing fresh blood or serum. The colony is small, transparent, shows beta hemolysis, and strong pathogenicity (Sriskandan and Slater 2006). The bacteria have strong resistance to the external environment and can survive for 6 days on a field at $29-33^{\circ} \mathrm{C}$. They are sensitive to disinfectants and can be killed quickly by common disinfectants (Gottschalk and Segura 2019).

\section{Epidemiology}

Sick, recessively infected and convalescent pigs after recovery are the main sources of infection. Recessively infected pigs normally carry bacteria in their tonsils and upper respiratory tract, so that they are the most dangerous source of infection. Pathogens can be detected in the nose, saliva, urine, blood, muscles, internal organs and joints of sick pigs. The disease can spread through direct contact with wounds. The respiratory tract and digestive tract are also the main routes of transmission of the disease. There is no strict age difference for this disease; both adult and young pigs can be infected, with the young pigs show the highest incidence (Aragon et al. 2012). The morbidity and fatality rates are very high. The chronic type of the disease is sporadic. There is no obvious seasonality for the disease, but the highest incidence is seen in summer and autumn, when the weather is hot and humid. A pandemic may occur from July to October. 


\section{Clinical symptoms}

Based on the length of the disease and clinical manifestations, swine streptococcal disease is divided into three types: acute sepsis, meningoencephalitis, and chronic lymph node abscess. Acute sepsis is common in the early stage of the epidemic. It has an acute onset and a short course. The symptoms include high fever (above $40^{\circ} \mathrm{C}$ ), depression, idle standing, loss of appetite, flushed conjunctiva with bleeding spots and tears, shortness of breath, dry nose with serous and purulent nasal fluid discharge. The skin on the neck, ears and lower abdomen becomes purple with bleeding spots. Most patients die within 3 to 5 days.

Meningoencephalitis is more common in suckling and weaning piglets, with meningoencephalitis as the main symptom. Other symptoms include constipation, runny or mucous nasal juices. After that, neurological symptoms appear quickly, such as blindly walking, unstable gait, moving in circles, screaming or twitching when touched, foaming at the mouth, strokes of the limbs, and death within 1 to 2 days.

Chronic lymph node abscesses are mostly transformed from acute form the disease. The symptoms include arthritis, endocarditis and tissue suppuration. It is characterized by mild disease, slow epidemic and long course, with some up to more than one month, and developmental delay. Polyarthritis is manifested by inflammation of one or more limb joints, swelling of the muscles around the joints, high limp, pain, and difficulty in standing. In severe cases, the hind limbs can be paralyzed, and finally death occurs due to physical failure and paralysis.

\section{Diagnosis}

A preliminary diagnosis can be made based on clinical symptoms and pathological changes. This disease should be distinguished from swine pneumonia, swine erysipelas and swine fever. Confirmation of preliminary diagnosis requires further laboratory tests such as microbiological examination. Different samples from the patients are taken according to different disease types, such as pus, joint fluid, liver, spleen, kidney, blood, lymph nodes, cerebrospinal fluid and brain tissue of sick or dead pigs to make smears and staining. By microscopic examination, diagnosis can be made if positive Gram, spherical or oval, and single, paired or chain-shaped cocci of different lengths are found (Alello et al. 2018).

\section{Prevention}

If the bacteria have been isolated, the most effective antibacterial drugs can be used for treatment following drug sensitivity test. If the bacteria have not been isolated and the disease is suspected, the drugs of choice are penicillin, tetracycline, oxytetracycline, and gentamicin. Immunization with vaccines is an important measure to prevent and control this disease. Swine streptococcal disease multivalent inactivated vaccine can be used
(Alello et al. 2018). A disinfection and isolation system can be established. The pig house should be kept clean, dry and ventilated, and a strict disinfection system should be established. Diagnosis of the disease should be made as soon as possible after discovering the epidemic. Sick pigs should be isolated, and the contaminated pig houses and utensils should be thoroughly cleaned and strictly disinfected.

\section{REFERENCES}

Acke E et al., 2004. Tetanus in the dog: Review and a case-report of concurrent Tetanus with Hiatal hernia. Irish Veterinary Journal 57(10): 593-597.

Al-Zamel F, 2012. Diagnosis of Mycobacterium tuberculosis. In: Understanding Tuberculosis - Global Experiences and Innovative Approaches to the Diagnosis. Pere-Joan Cardona (ed). IntechOpen.

Alello SE et al., 2018. The Merck Veterinary Manual. 11th Ed. Kenilworth, NJ: Merck \& Co., Inc.

Aragon V et al., 2012. Diseases of Swine. 1oth Ed. Jeffrey J Zimmerman et al (eds). John Wiley \& Sons, Inc.

Azami HY and Zinsstag J, 2018. Economics of Bovine Tuberculosis: A one health issue. In: Bovine Tuberculosis. M. Chambers et al (eds). Boston, MA: CABI.

Banyard AC and Fooks AR, 2020. Rabies life cycle, transmission and pathogenesis. In: Rabies and Rabies Vaccines. Hildegund C.J. (ed). Ertl. Springer, Cham.

Bapat PR et al., 2017. Prevalence of zoonotic Tuberculosis and associated risk factors in central Indian populations. Journal of Epidemiology and Global Health $7(4): 277-283$.

Beeler E and Ehnert K, 2020. Rabies in dogs and cats. In: Clinical Small Animal Internal Medicine. David S. Bruyette et al. (eds). John Wiley \& Sons, Inc.

Belœil PA et al., 2003. Listeria monocytogenes contamination of finishing pigs: An exploratory epidemiological survey in France. Veterinary Research 34(6): 737-748.

Bennett JE et al., 2014. Mandell, Douglas, and Bennett's Principles and Practice of Infectious Diseases. 9th Ed. John E. Bennett et al. (eds). Elsevier.

Bolton DC et al., 1982. Identification of a protein that purifies with the Scrapie Prion. Science 218: 1309-1311.

Bruce $\mathrm{M}$ et al., 1994. Transmission of Bovine Spongiform Encephalopathy and Scrapie to mice: Strain variation and the species barrier. Philosophical Transactions of the Royal Society of London. Series B, Biological sciences 343(1306): 405-411.

Bui $\mathrm{CM}$ et al., 2017. An overview of the epidemiology and emergence of Influenza A infection in humans over time. Archives of Public Health 75: 15.

Centers for Disease Control and Prevention, 2021. Rabies. https://www.cdc.gov/rabies/index.html (16-04-2021).

Cerqueira GM and Picardeau M, 2009. A century of Leptospira strain typing. Infection, Genetics and Evolution 9(5): 760-768.

Chlebicz A and Śliżewska K, 2018. Campylobacteriosis, Salmonellosis, Yersiniosis and Listeriosis as zoonotic 
foodborne diseases: A review. International Journal of Environmental Research and Public Health 15(5): 863.

D'Elia RV et al., 2019. Exploitation of the Bilosome Platform technology to formulate antibiotics and enhance efficacy of Melioidosis treatments. Journal of Controlled Release 298: 202-212.

Dallenga T et al., 2017. M. tuberculosis-induced necrosis of infected neutrophils promotes bacterial growth following phagocytosis by macrophages. Cell Host and Microbe 22(4): 519-530.

Ducrot $C$ et al., 2008. Review on the epidemiology and dynamics of BSE epidemics. Veterinary Research 39: 15.

Estes DM et al., 2010. Present and future therapeutic strategies for Melioidosis and glanders. Expert Review of Anti-Infective Therapy 8(3): 325-338.

Finke S and Conzelmann KK, 2005. Replication strategies of Rabies virus. Virus Research 111(2): 120-131.

Gilbert $\mathrm{M}$ et al., 2005. Cattle movements and Bovine Tuberculosis in Great Britain. Nature 435: 491-496.

Good MF, 2020. Streptococcus: An organism causing diseases beyond neglect. PLoS Neglected Tropical Diseases 14(5): eooo8095.

Gottschalk M and Segura M, 2019. Streptococcosis. In: Diseases of Swine. Jeffrey J. Zimmerman et al. (eds). John Wiley \& Sons, Inc.

Guedes IB et al., 2021. Usefulness of the ranking technique in the Microscopic Agglutination Test (MAT) to predict the most likely infecting serogroup of Leptospira. Frontiers in Veterinary Science 8: 654034 .

Haake DA and Levett PN, 2015. Leptospirosis in humans. Current Topics in Microbiology and Immunology. 387:65-97

Hampson K et al., 2015. Estimating the global burden of endemic canine Rabies. PLoS Neglected Tropical Diseases 9(4): eooo3709.

Kahn CM et al., 2007. The Merck/Merial Manual for Pet Health. 1st Ed., Westford, Massachusetts, U.S.A.: Merck \& Co., Inc.

Kariuki S et al., 2015. Antimicrobial resistance and management of invasive Salmonella disease. Vaccine 33(Supplement 3): C21-29.

Karpagam KB and Ganesh B, 2020. Leptospirosis: A neglected tropical zoonotic infection of public health importance-an updated review. European Journal of Clinical Microbiology and Infectious Diseases 39(5): 835-840.

Khan IA and Khan A, 2007. Prevalence and risk factors of Bovine Tuberculosis in Nili-Ravi buffaloes in the Punjab, Pakistan. Italian Journal of Animal Science, 6 (Supplement 2): 817-820.

Khan I et al., 2013. Glanders in animals: A review on epidemiology, clinical presentation, diagnosis and countermeasures. Transboundary and Emerging Diseases 6o(3): 204-221.

Khan MK et al., 2019. An overview on epidemiology of Tuberculosis. Mymensingh Medical Journal 28: 259-266.

Ladreyt $\mathrm{H}$ et al., 2020. Comparison of Japanese Encephalitis force of infection in pigs, poultry and dogs in Cambodian villages. Pathogens 9: 719.
Leake CJ, 1990. Japanese Encephalitis. Parasitology Today 6: 38 .

Lepe JA, 2020. Current aspects of Listeriosis. Medicina Clínica (English Edition) 154(11): 453-458.

Luca VC et al., 2012. Crystal structure of the Japanese Encephalitis virus envelope protein. Journal of Virology 86(4): 2337-2346.

Mahmood F et al., 2014. Molecular based epidemiology of bovine pulmonary tuberculosis-a mortal foe. Pakistan Veterinary Journal 34(2): 185-188.

Mansfield KL et al., 2017. Japanese Encephalitis virus infection, diagnosis and control in domestic animals. Veterinary Microbiology 201: 85-92.

Moustakas A et al., 2018. Abrupt events and population synchrony in the dynamics of Bovine Tuberculosis. Nature Communications 9: 2821.

Moustakas A and Evans MR, 2016. Regional and temporal characteristics of Bovine Tuberculosis of cattle in Great Britain. Stochastic Environmental Research and Risk Assessment 30: 989-1003.

Murillo A et al., 2020. Leptospira detection in cats in Spain by serology and molecular techniques. International Journal of Environmental Research and Public Health 17(5): 1600.

Peng $C$ et al., 2018. Molecular epidemiological survey and complete genomic phylogenetic analysis of $\mathrm{H} 6$ subtype Avian Influenza viruses in poultry in China from 2011 to 2016. Infection, Genetics and Evolution 65: 91-95.

Samy RP et al., 2017. Melioidosis: Clinical impact and public health threat in the tropics. PLoS Neglected Tropical Diseases 11: eooo4738.

Popoff MR, 2020. Tetanus in animals. Journal of Veterinary Diagnostic Investigation 32(2): 184-191.

Prusiner SB, 1991. Molecular biology of Prion diseases. Science 252(5012): 1512-1522.

Quan C et al., 2019. Avian Influenza A viruses among occupationally exposed populations, China, 20142016. Emerging Infectious Diseases 25(12): 2215-2225.

Rosa MI et al., 2017. IgM ELISA for Leptospirosis diagnosis: A systematic review and meta-analysis. Cien Saude Colet 22(12): 4001-4012.

Sriskandan S and Slater JD, 2006. Invasive disease and toxic shock due to zoonotic Streptococcus suis: An emerging infection in the East? PLoS Medicine 3: e187.

Taylor L and Nel L, 2015. Global epidemiology of canine Rabies: Past, present and future prospects. Veterinary Medicine: Research and Reports 6: 361-371.

Wallace RM et al., 2020. Role of oral Rabies vaccines in the elimination of dog-mediated human Rabies deaths. Emerging Infectious Diseases 26(12): 1-9.

World Health Organization, 2018. Rabies vaccines: WHO position paper, April 2018-Recommendations. Vaccine 36(37): 5500-5503.

Wu ZQ et al., 2017. Comparative epidemiology of human fatal infections with novel, high $\left(\mathrm{H}_{5} \mathrm{~N}_{6}\right.$ and $\left.\mathrm{H}_{5} \mathrm{~N}_{1}\right)$ and low $\left(\mathrm{H}_{7} \mathrm{~N}_{9}\right.$ and $\left.\mathrm{H}_{9} \mathrm{~N}_{2}\right)$ pathogenicity Avian Influenza A viruses. International Journal of Environmental Research and Public Health 14(3): 263.

Van ZKE et al., 2013. Glanders: An overview of infection in humans. Orphanet Journal of Rare Diseases 8: 131. 